\title{
Effects of Amyloid and Small Vessel Disease on White Matter Network Disruption
}

\author{
Hee Jin Kim ${ }^{\mathrm{a}}$, Kiho $\mathrm{Im}^{\mathrm{b}, *}$, Hunki Kwon ${ }^{\mathrm{c}}$, Jong Min Lee ${ }^{\mathrm{c}}$, Byoung Seok Ye ${ }^{\mathrm{d}}$, Yeo Jin Kimª \\ Hanna Cho ${ }^{\mathrm{e}}$, Yearn Seong Choe ${ }^{\mathrm{f}}$, Kyung Han Lee ${ }^{\mathrm{f}}$, Sung Tae Kim ${ }^{\mathrm{g}}$, Jae Seung Kim ${ }^{\mathrm{h}}$, Jae Hong Lee ${ }^{\mathrm{i}}$, \\ Duk L. Na ${ }^{\mathrm{a}}$ and Sang Won $\mathrm{Seo}^{\mathrm{a}, *}$ \\ ${ }^{a}$ Department of Neurology, Samsung Medical Center, Sungkyunkwan University School of Medicine, Seoul, Korea \\ ${ }^{\mathrm{b}}$ Division of Newborn Medicine, Boston Children's Hospital, Harvard Medical School, Boston, MA, USA \\ ${ }^{\mathrm{c}}$ Department of Biomedical Engineering, Hanyang University, Seoul, Korea \\ ${ }^{\mathrm{d}}$ Department of Neurology, Yonser University College of Medicine, Seoul, Korea \\ e Department of Neurology, Gangnam Severance Hospital, Yonsei University College of Medicine, Seoul, Korea \\ ${ }^{\mathrm{f}}$ Department of Nuclear Medicine, Samsung Medical Center, Sungkyunkwan University School of Medicine, \\ Seoul, Korea \\ ${ }^{\mathrm{g}}$ Department of Radiology, Samsung Medical Center, Sungkyunkwan University School of Medicine, Seoul, Korea \\ ${ }^{\mathrm{h}}$ Department of Nuclear Medicine, University of Ulsan College of Medicine, Asan Medical Center, Seoul, Korea \\ ${ }^{\mathrm{i}}$ Department of Neurology, University of Ulsan College of Medicine, Asan Medical Center, Seoul, Korea
}

Handling Associate Editor: YongSoo Shim

Accepted 3 October 2014

\begin{abstract}
There is growing evidence that the human brain is a large scale complex network. The structural network is reported to be disrupted in cognitively impaired patients. However, there have been few studies evaluating the effects of amyloid and small vessel disease (SVD) markers, the common causes of cognitive impairment, on structural networks. Thus, we evaluated the association between amyloid and SVD burdens and structural networks using diffusion tensor imaging (DTI). Furthermore, we determined if network parameters predict cognitive impairments. Graph theoretical analysis was applied to DTI data from 232 cognitively impaired patients with varying degrees of amyloid and SVD burdens. All patients underwent Pittsburgh compound-B (PiB) PET to detect amyloid burden, MRI to detect markers of SVD, including the volume of white matter hyperintensities and the number of lacunes, and detailed neuropsychological testing. The whole-brain network was assessed by network parameters of integration (shortest path length, global efficiency) and segregation (clustering coefficient, transitivity, modularity). PiB retention ratio was not associated with any white matter network parameters. Greater white matter hyperintensity volumes or lacunae numbers were significantly associated with decreased network integration (increased shortest path length, decreased global efficiency) and increased network segregation (increased clustering coefficient, increased transitivity, increased modularity). Decreased network integration or increased network segregation were associated with poor performances in attention, language, visuospatial, memory, and frontal-executive functions. Our results suggest that SVD alters white matter network integration and segregation, which further predicts cognitive dysfunction.
\end{abstract}

Keywords: Amyloid, diffusion tensor imaging, graph theory, small vessel disease, white matter network

\footnotetext{
${ }^{*}$ Correspondence to: Kiho Im, PhD, Division of Newborn Medicine, Boston Children's Hospital, 1 Autumn St. Boston, MA 02115, USA. Tel.: +1 857218 5142; Fax: +1 671730 4671; E-mail: Kiho.Im@childrens.harvard.edu. and Sang Won Seo, MD,
}

$\mathrm{PhD}$, Department of Neurology, Sungkyunkwan University School of Medicine, Samsung Medical Center, 50 Ilwon-dong, Kangnamku, Seoul 135-710, Republic of Korea. Tel.: +82 23410 1233; Fax: +82 23410 0052; E-mail: sangwonseo@empal.com. 


\section{INTRODUCTION}

The most common causes of dementia are mixed types of dementia, comprising features of Alzheimer's disease (AD) and small vessel disease (SVD) [1]. Amyloid burdens and SVD markers such as white matter hyperintensities (WMH) and lacunes are reported to be independently associated with cognitive impairments [2]. Recent studies also showed that amyloid and SVD burdens independently affected cortical atrophy in specific patterns, and had differential effects on functional networks $[3,4]$. However, there were few studies evaluating the independent effects of amyloid and SVD burdens on microstructural change of white matter $[5,6]$.

Diffusion tensor imaging (DTI) is a noninvasive technique that can be used to reflect the microstructural tissue status. Recent advance in graph theoretical analysis provides an efficient and quantitative way to model a large-scale complex network. Using the theoretical framework of networks and graphs, the brain can be represented as set of nodes (brain regions) joined in pairs by lines (white matter connectivity) [7]. The ability to rapidly combine specialized information from distributed nodes can be quantified into measures of network integration (shortest path length, global efficiency), and the ability for specialized processing to occur within densely interconnected groups of nodes can be quantified into measures of network segregation (clustering coefficient, transitivity, modularity) [8].

There is growing evidence that the human brain is a large scale complex network [9-11]. The ideal healthy brain exhibits a small-world character [12], which optimally balances information segregation and integration, resulting in efficient organization that reduces the cost of maintaining many connections but also allows for efficient movement of information [13]. The efficient organization of these networks is affected in various degenerative diseases [14-16]. Furthermore, recent studies suggested that network disruption follows disease-specific patterns that resemble the architecture of brain connectivity networks $[10,11]$. However, the relationship between proteinopathies in these degenerative diseases, such as amyloid- $\beta$ in $\mathrm{AD}$, and brain networks remains unclear. In addition, there have been few studies evaluating the effects of SVD markers on large-scale brain networks [17]. Furthermore, there were no studies evaluating whether amyloid burdens and SVD markers (WMH and lacunes) were independently associated with white matter network, which were further associated with cognitive impairments.
In this study, we aimed to determine if amyloid and SVD burdens independently affect the whole-brain white matter networks by applying graph analysis to DTI data in a large sample of patients with varying degrees of amyloid and SVD burden. The whole-brain network was assessed by network parameters of integration (shortest path length, global efficiency) and segregation (clustering coefficient, transitivity, modularity). We also examined if network parameters predict cognitive impairment.

\section{MATERIALS AND METHODS}

\section{Participants}

We prospectively recruited 251 subjects with cognitive impairment who underwent Pittsburgh compound-B (PiB)-PET and structural brain MRI. We included 45 patients with amnestic mild cognitive impairment (aMCI), 69 with probable AD dementia, 67 with subcortical vascular MCI (svMCI), and 70 with subcortical vascular dementia (SVaD). All patients were clinically diagnosed at the Samsung Medical Center. Probable AD dementia patients fulfilled criteria proposed by the National Institute of Neurological and Communicative Disorders and Stroke and the AD and Related Disorders Association [18]. Patients with SVaD met the diagnostic criteria for vascular dementia as determined by the Diagnostic and Statistical Manual of Mental Disorders-Fourth Edition (DSM-IV) and fulfilled imaging criteria for SVaD proposed by Erkinjuntti et al. [19]. The aMCI and svMCI patients met Petersen's criteria for MCI with modifications as previously described [20]. All svMCI and SVaD patients had severe WMH on their MRI scans, which was defined as a cap or band (periventrivular $\mathrm{WMH}, \mathrm{PWMH}) \geq 10 \mathrm{~mm}$ and deep white matter lesions (deep WMH, DWMH) $\geq 25 \mathrm{~mm}$, as modified from the Fazekas ischemia criteria [21]. All aMCI and $\mathrm{AD}$ cases were classified as having minimal (PWMH $<5 \mathrm{~mm}$ and DWMH $<5 \mathrm{~mm}$ ) or moderate WMH (between minimal and severe grades). The WMH were in compliance with the WMH of presumed vascular origin definition proposed by the Standards for Reporting Vascular Changes on Neuroimaging (STRIVE) [22]. Detailed criteria for aMCI and svMCI are described in Supplementary Text 1. Patients with aMCI or AD were regarded as having AD-related cognitive impairment (ADCI), while those with svMCI or SVaD were considered to have subcortical vascular cognitive impairment (SVCI). We excluded patients with territorial infarctions and those 
with WMH due to radiation injury, multiple sclerosis, vasculitis, or leukodystrophy. All patients underwent a detailed diagnostic process including taking medical history, semi-structured questionnaires with respect to cognitive, behavioral, and functional impairments, and neurological examination [23]. Blood tests included a complete blood count, blood chemistry test, vitamin $\mathrm{B}_{12} /$ folate measurements, syphilis serology, thyroid function testing, and APOE genotyping. We calculated disease duration from symptom onset to date of PiB-PET scan (see Table 1).

We obtained written informed consent from each patient and the Institutional Review Board of the Samsung Medical Center approved the study protocol.

\section{PET acquisition}

$\left[{ }^{11} \mathrm{C}\right]$ PiB-PET scanning was performed at Samsung or Asan Medical Center using a Discovery STe PET/CT scanner (GE Medical Systems, Mil- waukee, WI) in a 3-dimensional scanning mode that examined 35 slices of 4.25 -mm thickness spanning the entire brain. Detailed methods are described in Supplementary Text 2.

\section{PiB-PET data analysis}

PiB-PET images were co-registered to individual MRIs, which were normalized to a T1-weighted MRI template. The quantitative regional values of $\mathrm{PiB}$ retention on the spatially normalized $\mathrm{PiB}$ images were obtained by using an automated VOI (voxel of interest) analysis using the automated anatomical labeling (AAL) atlas. Data processing was performed using SPM version 5 (SPM5) under Matlab 6.5 (Mathworks, Natick, MA).

Detailed methods for the calculation of global PiB retention ratios are described in Supplementary Text 3. We defined $\mathrm{PiB}$ retention ratio as a continuous variable representing amyloid burden.

Table 1

Demographics, clinical characteristics, and imaging MRI markers of study participants

\begin{tabular}{|c|c|c|c|c|c|c|c|}
\hline \multirow[t]{2}{*}{ Group } & \multirow[t]{2}{*}{ Total } & \multicolumn{3}{|c|}{ ADCI } & \multicolumn{3}{|c|}{ SVCI } \\
\hline & & Subtotal & aMCI & $\mathrm{AD}$ & Subtotal & svMCI & SVaD \\
\hline Number & 232 & 107 & 43 & 64 & 125 & 59 & 66 \\
\hline \multicolumn{8}{|l|}{ Demographics } \\
\hline Age, y & $72.1(8.1)$ & $69.9(8.8)^{*}$ & $70.1(7.9)$ & $69.7(9.5)$ & $73.9(6.9)$ & $74.1(6.6)$ & $73.7(7.2)$ \\
\hline Gender, female, n (\%) & $131(56.5)$ & $60(56.1)$ & $20(46.5)$ & $40(62.5)$ & $71(56.8)$ & $34(57.6)$ & $37(56.1)$ \\
\hline Education, $\mathrm{y}$ & $10.1(5.4)$ & $11.2(5.4)^{*}$ & $12.4(4.7)$ & $10.4(5.7)$ & $9.2(5.2)$ & $9.8(5.4)$ & $8.7(5.1)$ \\
\hline \multicolumn{8}{|l|}{$\begin{array}{l}\text { Cardiovascular risk } \\
\text { factors, } \mathrm{n}(\%)\end{array}$} \\
\hline Hypertension & $144(62.1)$ & $50(46.7)^{*}$ & $16(37.2)$ & $34(53.1)$ & $94(75.2)$ & $43(72.9)$ & $51(77.3)$ \\
\hline Diabetes mellitus & $47(20.3)$ & $15(14.0)^{*}$ & $5(11.6)$ & $10(15.6)$ & $32(25.6)$ & $16(27.1)$ & $16(24.2)$ \\
\hline Hyperlipidemia & $68(29.3)$ & $25(23.4)$ & $10(23.3)$ & $15(23.4)$ & $43(34.4)$ & $18(30.5)$ & 25 (37.9) \\
\hline Heart disease & $34(14.7)$ & $12(11.2)$ & $7(16.3)$ & $5(7.8)$ & $22(17.6)$ & $16(27.1)$ & $6(9.1)$ \\
\hline \multicolumn{8}{|l|}{$A P O E$ genotype, $\mathrm{n}(\%)^{\dagger}$} \\
\hline$\varepsilon 2$ allele carrier & $18 / 225(8.0)$ & $6 / 103(5.8)$ & $3 / 41(7.3)$ & $3 / 62(4.8)$ & $12 / 122(9.8)$ & $7 / 59(11.9)$ & $5 / 63(7.9)$ \\
\hline$\varepsilon 4$ allele carrier & $82 / 225(36.4)$ & $49 / 103(47.6)^{*}$ & $16 / 41(39.0)$ & $33 / 62(53.2)$ & $33 / 122(27.0)$ & $14 / 59(23.7)$ & $19 / 63(30.2)$ \\
\hline \multicolumn{8}{|l|}{ Small vessel MRI markers } \\
\hline WMH volume, $\mathrm{ml}$ & $22.9(22.3)$ & $4.3(5.6)^{*}$ & $3.2(3.2)$ & $5.1(6.7)$ & $38.8(18.7)$ & $33.8(17.9)$ & $43.2(18.5)$ \\
\hline Lacunes, $\mathrm{n}$ & $6.7(12.1)$ & $0.4(1.5)^{*}$ & $0.6(2.2)$ & $0.3(0.8)$ & $12.2(14.3)$ & $7.5(8.5)$ & $16.4(17.0)$ \\
\hline $\begin{array}{l}\text { Global } \mathrm{PiB} \text { global } \\
\text { retention ratio }\end{array}$ & $1.8(0.5)$ & $2.1(0.5)^{*}$ & $1.8(0.5)$ & $2.2(0.4)$ & $1.5(0.4)$ & $1.5(0.4)$ & $1.6(0.5)$ \\
\hline MMSE & $22.4(5.7)$ & $21.3(6.4)^{*}$ & $25.4(4.7)$ & $18.5(5.8)$ & $23.3(4.9)$ & $26.4(2.8)$ & $20.6(4.9)$ \\
\hline CDR-SOB & $3.8(3.3)$ & $3.7(2.7)$ & $1.6(1.0)$ & $5.1(2.5)$ & $3.9(3.8)$ & $1.4(1.1)$ & $6.3(3.8)$ \\
\hline Disease duration, months & $53.8(29.7)$ & $51.9(26.7)$ & $42.4(24.5)$ & $58.2(26.4)$ & $55.3(32.1)$ & $46.3(25.6)$ & $63.4(35.2)$ \\
\hline \multicolumn{8}{|l|}{ Network parameters } \\
\hline shortest path length & $1.22(0.08)$ & $1.19(0.06)$ & $1.18(0.04)$ & $1.20(0.07)$ & $1.25(0.09)$ & $1.22(0.08)$ & $1.27(0.09)$ \\
\hline global efficiency & $0.89(0.03)$ & $0.90(0.02)$ & $0.90(0.02)$ & $0.90(0.03)$ & $0.87(0.03)$ & $0.88(0.03)$ & $0.87(0.03)$ \\
\hline clustering coefficient & $3.96(0.85)$ & $3.52(0.55)$ & $3.48(0.44)$ & $3.54(0.61)$ & $4.34(0.88)$ & $4.02(0.77)$ & $4.62(0.88)$ \\
\hline transitivity & $3.53(0.76)$ & $3.12(0.46)$ & $3.05(0.36)$ & $3.17(0.52)$ & $3.88(0.78)$ & $3.59(0.73)$ & $4.14(0.74)$ \\
\hline modularity & $0.53(0.05)$ & $0.51(0.04)$ & $0.51(0.03)$ & $0.51(0.04)$ & $0.55(0.05)$ & $0.54(0.05)$ & $0.57(0.04)$ \\
\hline
\end{tabular}

aMCI, amnestic mild cognitive impairment; AD, Alzheimer's disease; ADCI, Alzheimer's disease related cognitive impairment; SVCI, subcortical vascular cognitive impairment; svMCI, subcortical vascular mild cognitive impairment; SVaD, subcortical vascular dementia; APOE, Apolipoprotein E; WMH, white matter hyperintensities; PiB, Pittsburgh compound B; MMSE, Mini-Mental State Examination; CDR-SOB, clinical dementia rating-sum of boxes. ${ }^{*} P<0.05$ in the chi-square test or independent t-test comparing ADCI and svMCI. ${ }^{\dagger}$ APOE genotyping was performed in 225 out of 232 participants. Values are expressed as mean (standard deviation) or number (\%). 


\section{MR imaging techniques}

Three dimensional fluid-attenuated inversion recovery (FLAIR) and DTI images were acquired from all subjects at the Samsung Medical Center using the same 3.0T MRI scanner (Philips 3.0T Achieva). In all subjects, these images were obtained in one session and all MR images were obtained in the same orientation and slice positions. Detailed imaging parameters are described in Supplementary Text 4. For whole-brain DT-MRI examinations, sets of axial diffusion-weighted single-shot echo-planar images were collected with the following parameters: $128 \times 128$ acquisition matrix, $1.72 \times 1.72 \times 2 \mathrm{~mm}^{3}$ voxel size, 70 axial slices with a $22 \times 22 \mathrm{~cm}^{2}$ field of view, echo time (TE) $60 \mathrm{~ms}$, repetition time (TR) $7696 \mathrm{~ms}$; flip angle $90^{\circ}$; slice gap $0 \mathrm{~mm}$; b-factor of $600 \mathrm{smm}^{-2}$. Baseline images were without weighting $[0,0,0]$ and diffusion-weighted images were acquired from 45 different directions. All axial sections were acquired parallel to the anterior commissureposterior commissure line and perpendicular to the mid-sagittal plane.

\section{Measurement of regional WMH volume}

We quantified WMH volume (in ml) on FLAIR images using an automated method as previously described [20]. First, we extracted the WMH candidate regions using T1-weighted images to avoid misclassification in the subarachnoid space and cerebrospinal fluid interface, which cannot be excluded by intensity threshold or the conventional brain extraction tools. Second, in order to extract WMH, a threshold method was applied to the masked FLAIR MRI (the regions of white matter and gray matter in FLAIR images). Even though the threshold value was selected considering the range of image intensities, segmented results could contain false positive or false negative regions depending on the extent of WMH. The rate of agreement between two neurologists was $92.3 \%$. If the results contained an error, the threshold value was reselected through visual inspection by two raters, and they reached a consensus in the case of discrepancy.

\section{Assessment of lacunes on MRI}

A lacune was defined as a lesion $\geq 3 \mathrm{~mm}$ and $\leq 15 \mathrm{~mm}$ in diameter with low signal on $\mathrm{T} 1$-weighted images, high signal on T2-weighted images, and perilesional halo on FLAIR images. This is in compliance with the definition for a lacune of presumed vascular origin as recommended by STRIVE [22]. Two expe- rienced neurologists blinded to the clinical patient data reviewed the number and location of the lacunes on 80 axial slices of FLAIR imaging. The kappa value between the two neurologists for the presence of lacunes was 0.78 and a consensus was reached in any case of discrepancy.

\section{Network node definition}

We used the AAL atlas [24] to parcellate the whole cerebral cortex into 78 areas (39 regions in each hemisphere) and define the nodes of the brain graph. Individual T1-weighted images were nonlinearly registered to the ICBM152 T1 template in the MNI space [25]. The AAL atlas was transformed from the MNI space to the T1 native space using the inverse transformation with a nearest-neighbor interpolation method.

\section{Network edge definition}

Distortions in diffusion tensor images caused by eddy currents and simple head motions were corrected by the diffusion toolbox of the FSL package (http://www.fmrib.ox.ac.uk/fsl/fdt). Diffusion tensor models were estimated and the fractional anisotropy (FA) and mean diffusivity (MD) were calculated at each voxel. We reconstructed whole-brain white matter fiber tracts in native diffusion space for each subject using the fiber assignment by continuous tracking algorithm [26] embedded in the Diffusion Toolkit (http://www.trackvis.org/) [27]. We terminated tracking when the angle between two consecutive orientation vectors was greater than the given threshold of $45^{\circ}$ or when both ends of the fibers extended outside of the white matter mask which was generated by the tissue segmentation process [28]. Fiber cutoff filter was applied so that noisy short and false-positive long fibers (shorter than $20 \mathrm{~mm}$ and longer than $200 \mathrm{~mm}$ ) were filtered out [29].

T1-weighted images were co-registered to the b0 images using the affine registration tool from the FSL package (http://www.fmrib.ox.ac.uk/fsl/flirt). Reconstructed whole-brain fiber tracts were inversely transformed into the $\mathrm{T} 1$ space and fiber tracts and AAL-based parcellated regions were located in the same space. Two nodes (regions) were considered to be structurally connected by an edge when at least the end points of three fiber tracts were located in these two regions. A threshold of the number of fiber tracts was selected to reduce the risk of false-positive connections due to noise or limitations in the deterministic tractography $[28,30]$. FA value is an important index 
to evaluate fiber integrity [31]. In this study, mean FA value along all fibers connecting a pair of regions was used to weight the edge. Finally, weighted structural networks represented by symmetric $78 * 78$ matrices were constructed for each individual.

\section{Network analysis}

Graph theoretical analyses were carried out on weighted connectivity networks using the Brain Connectivity Toolbox (http://www.brain-connectivitytoolbox.net) [8]. To measure network integration, we calculated the average shortest path length between all pairs of nodes in the network. This is known as the characteristic path length, L, of the network [32], which is computed as follows:

$$
L=\frac{1}{N} \sum_{i=1}^{N} \frac{\sum_{j \neq i}^{N} L_{i j}}{N-1}
$$

In addition, the global efficiency, $E$, was computed as the average inverse shortest path length [13].

$$
E=\frac{1}{N} \sum_{i=1}^{N} \frac{\sum_{j \neq i}^{N} L_{i j}^{-1}}{N-1},
$$

where $N$ is the number of nodes and $L_{i j}$ is the shortest weighted path length between node $i$ and $j$.

We calculated the weighted clustering coefficient, $C$, and transitivity, $T$, as measures of network segregation [33], which were computed as follows:

$$
\begin{aligned}
C & =\frac{1}{N} \sum_{i=1}^{N} \frac{2 t_{i}}{k_{i}\left(k_{i}-1\right)}, \\
t_{i} & =\frac{1}{2} \sum_{j, h \in N}\left(w_{i j} w_{i h} w_{j h}\right)^{1 / 3}, \\
T & =\frac{\sum_{i=1}^{N} 2 t_{i}}{\sum_{i=1}^{N} k_{i}\left(k_{i}-1\right)},
\end{aligned}
$$

where $k_{i}$ is the degree of a node $i$ (the number of links connected to a node $i$ ), $w$ is a connection weight, and $t_{i}$ is the weighted geometric mean of triangles around $i$.

The graph measures were scaled against the mean values of graph measures obtained from 1,000 matched random graphs that preserved the same number of nodes, edges, and degree sequence [34].

A brain graph can generally be subdivided or partitioned into modules of nodes. A module is defined as a group of nodes that have strong connections to other nodes within the module but weak connections to nodes outside the module. Optimal modular structures and modularity values were estimated by maximizing the ratio of within-modular to between-modular edges with optimization algorithms, and the number of modules was measured [35].

Of 251 subjects, we excluded six patients who failed in WMH volume measurement and 13 patients whose quality of diffusion image (low signal-to-noise ratio) was not sufficient to reconstruct reliable fiber tracts. Finally, 232 subjects (43 aMCI, 64 AD, 59 svMCI, and $66 \mathrm{SVaD}$ ) were analyzed in this study.

\section{Cortical thickness data analysis}

T1-weighted images were processed using the standard MNI anatomic pipeline. Further image processing for cortical thickness measurements is described in Supplementary Text 5.

\section{Neuropsychological tests}

A total of 230 subjects out of 232 participants underwent neuropsychological tests using a standardized neuropsychological battery [36, 37]. The battery contains digit span (forward and backward), Boston Naming Test, Rey-Osterrieth Complex Figure Test (RCFT; copying, immediate and 20-min delayed recall, and recognition), Seoul Verbal Learning Test (SVLT; 3 learning-free recall tests of 12 words, 20-min delayed recall test for those 12 items, and a recognition test), a phonemic and semantic Controlled Oral Word Association Test (COWAT), and Stroop Test (word and color reading of 112 items during a 2-min period).

\section{Statistical analysis}

To evaluate the association between neuroimaging markers ( $\mathrm{PiB}$ retention ratio, WMH volume, and number of lacunes) and network parameters, multiple linear regression analysis was performed. In model 1 , we entered age, gender, education, $\mathrm{PiB}$ retention ratio, WMH volume, and number of lacunes to find the independent effects of each imaging marker. In model 2, we additionally entered mean cortical thickness as an independent variable. During the analysis, we tested for assumption of multiple regression such as normality and homoscedasticity, which revealed to be non-violated.

To evaluate the association between network parameters and neuropsychological results, multiple linear 


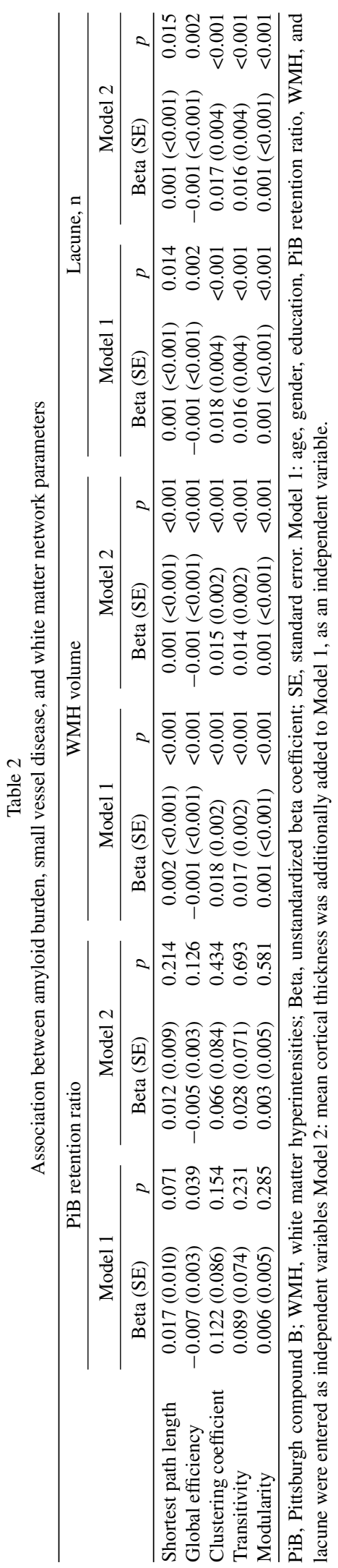


regression analysis was performed. In model 1 , we entered age, gender, education, $\mathrm{PiB}$ retention ratio, WMH volume, number of lacunes, and each network parameter as independent variables and each neuropsychological result score as dependent variables. In model 2 , mean cortical thickness was additionally entered as an independent variable. False discovery rate correction was performed to correct for multiple comparisons of 14 tests in the neuropsychological test battery.

We also performed subgroup analyses in ADCI (aMCI or AD) and SVCI (svMCI or SVaD) to evaluate the association between neuroimaging markers and network parameters and between network parameters and neuropsychological results in each group.

\section{RESULTS}

\section{Demographics}

Our participants had a mean age of 72.1 years and had varying degrees of SVD and amyloid burden (Table 1). The neuropsychological test results of the participants are shown in Supplementary Table 1.

\section{PiB retention ratio, SVD markers, and network parameters}

In both model 1 and model 2, greater WMH volumes were significantly associated with disrupted network integration, indicated by increased shortest path length and decreased global efficiency; and increased network segregation, indicated by increased clustering coefficient, increased transitivity, and increased modularity (Table 2). Likewise, a greater number of lacunes was significantly associated with disrupted network integration and increased network segregation (Table 2). Fig. 1 shows the significant correlations between SVD markers and network parameters. In model 1, greater $\mathrm{PiB}$ retention ratios were significantly associated with decreased global efficiency. However, in model 2, PiB retention ratios were not associated with any measures of network parameters (Table 2).

Subgroup analyses showed that in both ADCI and SVCI subgroups, SVD but not $\mathrm{PiB}$ retention ratios were associated with altered network parameters (Supplementary Table 2).

\section{Network parameters and neuropsychological results}

In model 1, we observed significant correlations between network parameters and cognitive performance as shown in Table 3. Increased shortest path length was associated with poor performance in digit span backward, RCFT copy, RCFT delayed recall, SVLT immediate/delayed recall, COWAT supermarket, and Stroop color reading. Decreased global efficiency was associated with poor performance in digit span backward, RCFT copy, RCFT delayed recall, SVLT immediate/delayed recall, and COWAT supermarket. Increased clustering coefficient was associated with poor performance in digit span backward, BNT, RCFT copy, RCFT delayed recall, SVLT immediate recall, COWAT animal/phonemic, and Stroop color reading. Increased transitivity was associated with poor performance in digit span backward, BNT, RCFT copy, RCFT immediate/delayed recall, SVLT immediate/delayed recall, COWAT ani$\mathrm{mal} /$ supermarket/phonemic, and Stroop color reading. Increased modularity was associated with poor performance in RCFT copy.

In model 2 , increased shortest path length remained to be associated with poor performance in digit span backward, RCFT copy, and SVLT delayed recall; decreased global efficiency remained to be associated with poor performance in SVLT delayed recall; and decreased clustering coefficient, transitivity, and modularity remained to be associated with poor performance in RCFT copy. However, the significant association between network parameters and performances in other cognitive domains disappeared.

Subgroup analyses also showed that in both ADCI and SVCI groups, when cortical thickness was additionally controlled, the significant association between network parameters and cognitive performances disappeared in most domains (Supplementary Table 3).

\section{DISCUSSION}

This study analyzed DTI data using graph theoretical analysis to examine alterations in white matter networks related to amyloid and SVD burdens in cognitively impaired patients. Our main findings were as follows: 1) Greater WMH volume or number of lacunes were associated with decreased network integration and increased network segregation; and 2) decreased network integration or increased network segregation correlated with decreased neuropsychological performance in variable domains including attention, language, visuospatial, memory, and frontalexecutive functions regardless of amyloid or SVD burdens. Taken together, our findings suggested that SVD burdens are associated with an imbalance in the 


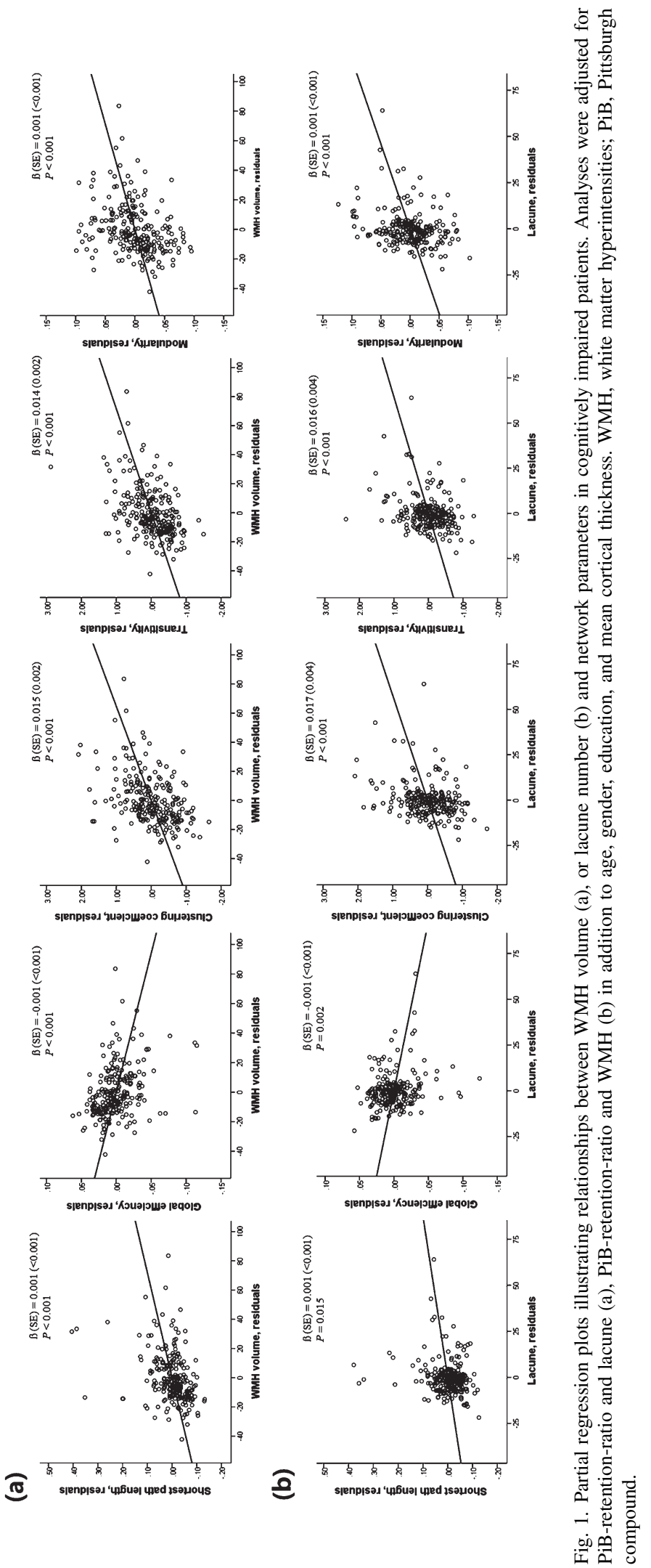




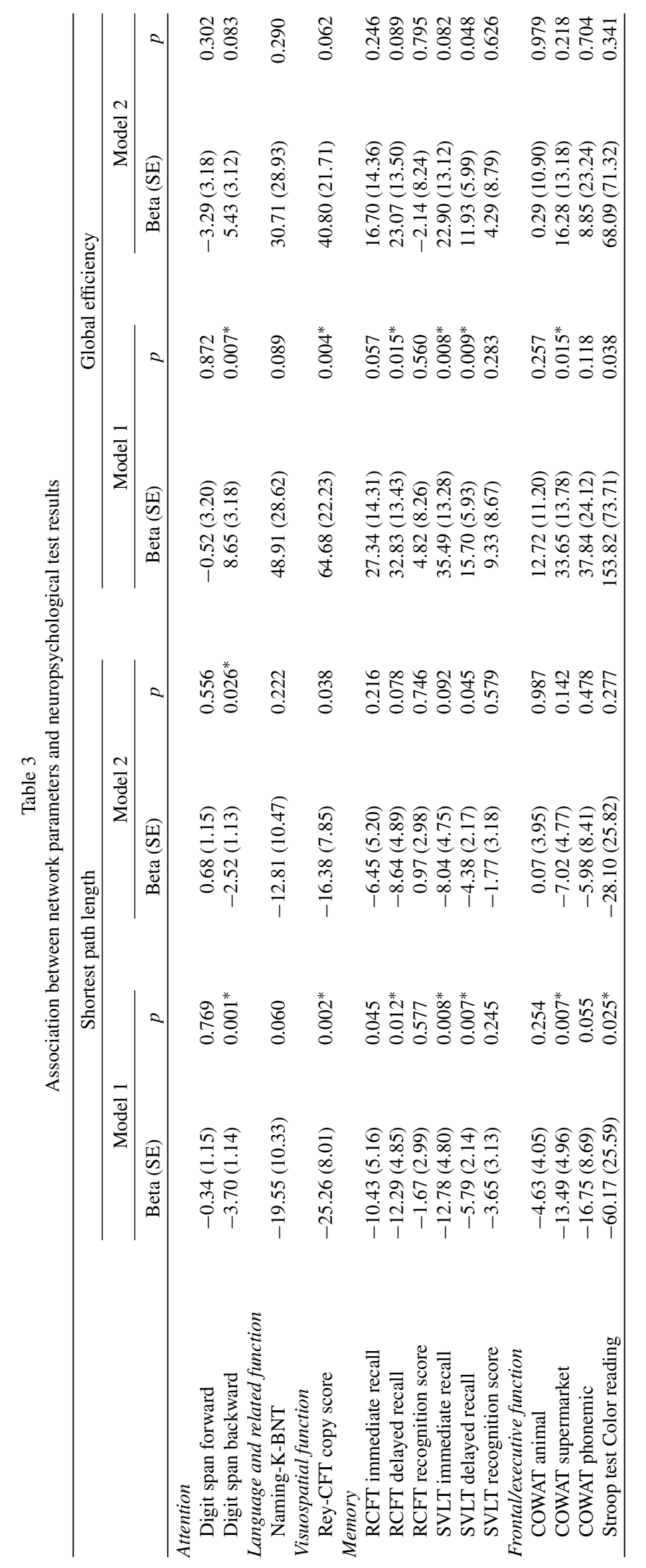




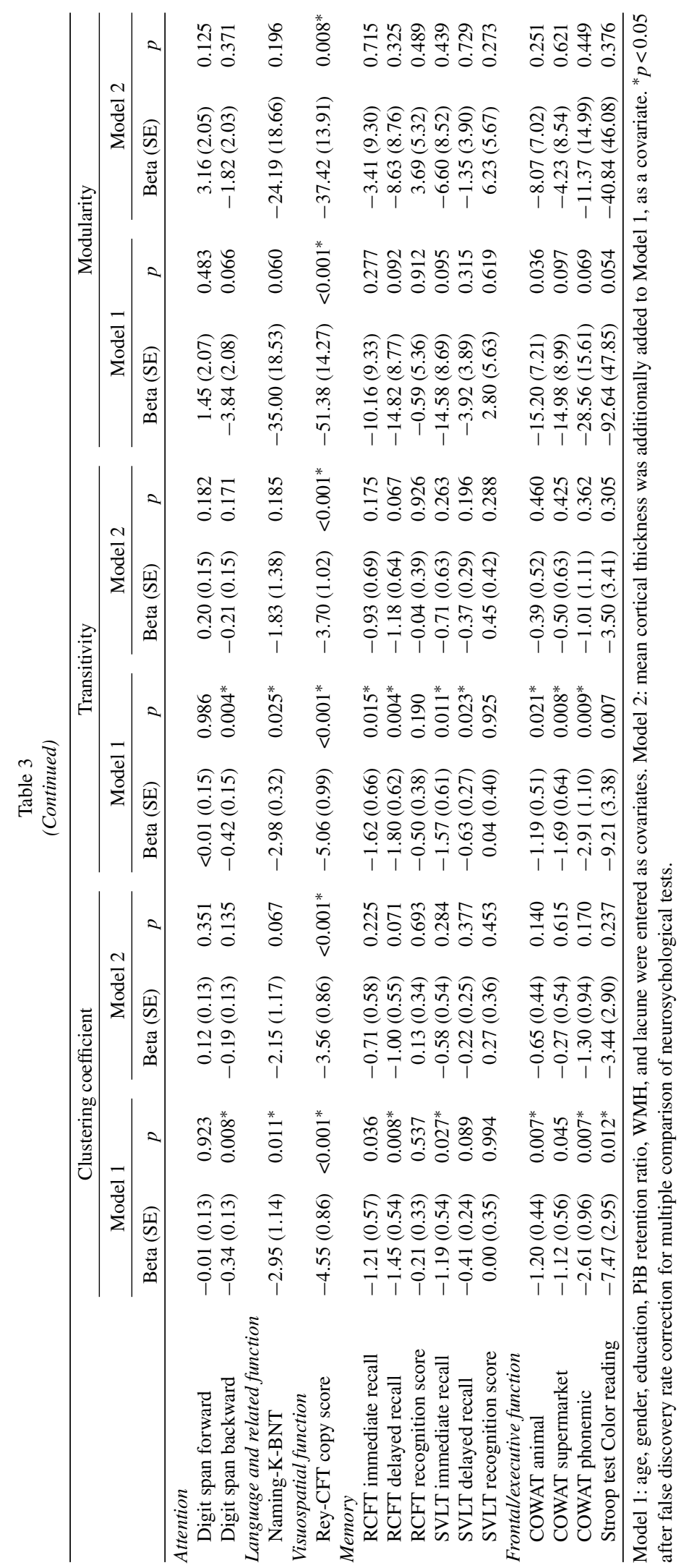


large-scale brain networks which leads to cognitive deficits.

Our major finding was that SVD burdens alter the white matter network toward decreased network integration and increased network segregation, which is characterization of 'regular' topology. Regular topology has increased short-range connections but decreased long-range connections, eventually resulting in inefficient organization [13]. Our findings suggested that altered network patterns induced by SVD burdens might be different from those shown in $\mathrm{AD}$, in which white matter network is imbalanced toward a more 'random' topology, as indicated by decreased network segregation and increased network integration [38]. Although several studies have shown the relationships between SVD markers and white matter injury [5, 6], our study is notable because this result shows the effect of SVD on changes in network characters, independent of amyloid and cortical atrophy.

The pathobiology of altered networks induced by SVD burdens is important. Regarding decreased integration of network, it might be explained to the fact that ischemia, usually located in periventricular or deep white matter areas, causes demyelination and axonal loss of long projection fibers, resulting in reduced global efficiency of signal propagation [39, 40]. Regarding increased network segregation, there are several possible interpretations. First, the increased network segregation might be related to some changes in microstructure and white matter connectivity in hub regions in particular. The loss or damage of hubs is likely to fragment a network into disconnected parts, resulting in increased network segregation [41]. Second, it might not stem from an actual higher segregation but an artifact arising from relative decrease in network integration [28].

We found that amyloid burdens were not associated with any white matter network parameters. Previous studies regarding structural network in AD patients show inconsistent results, as some reported increased network integration [38], while others reported decreased network integration [16]. However, to our knowledge, there have been no studies investigating alterations of white matter network according to 'amyloid- $\beta$ burden' in particular. It is possible that in previous studies of $\mathrm{AD}$, not amyloid burden itself, but other factors characterizing AD might have been responsible for altered white matter network.

Our final major finding was that decreased network integration or increased network segregation correlated with decreased neuropsychological performance in variable domains including attention, language, visuospatial, memory, and frontal-executive functions regardless of amyloid and SVD burdens. There are several clinical implications. First, altered network toward a 'regular' topology predicts worse performances in cognition. Previous studies regarding the relationship between white matter network parameters and cognition have shown that decreased network integration and decreased network segregation were related to cognitive impairments in AD patients [16, 42]. Interestingly, our findings suggested that altered network toward a 'regular' topology is not only a characteristic of a network driven by SVD burdens but also the predictor of cognitive impairments in these patients. Second, multiple cognitive domains were related to white matter network parameters. Frontal-executive dysfunction by white matter lesions has been largely studied [43]. Recent approaches on graph theoretical analysis have shown that white matter network parameters are associated with memory and frontal dysfunctions [16, 42]. Our data additionally shows that visuospatial dysfunction is strongly associated with altered white matter network parameters. Third, it is noteworthy that most of the association between altered white matter network and cognitive profiles were not independent of cortical atrophy, which suggests that cortical atrophy might have mediated the effect of white matter network disruption on cognitive impairment in various domains. Meanwhile, the association between increased network segregation and visuospatial dysfunction was not modulated by cortical thickness. This provides a unique clue that the network of white matter and thinning of grey matter have complex relationships and have different roles leading to cognitive impairment according to their respective cognitive domains. The complex relationships between amyloid, SVD, white matter network, cortical atrophy, and cognition need to be further studied. Finally, network parameters independently affect cognition regardless of amyloid and SVD burdens. A recent study based on AD patients showed that disturbances in white matter connectivity explained a substantial proportion of the variance in cognitive function on top of markers of brain atrophy and SVD [42]. Along with previous studies, our findings suggest that white matter network parameters may explain a substantial portion of the variation in cognitive impairments in these subjects. Longitudinal studies are necessary to further validate our suggestion in individual subjects.

There are several limitations in our study. First, we used a single diffusion tensor-based deterministic tractography algorithm, which does not detect fiber 
crossings. More recent acquisition sequences, such as the high angular resolution diffusion imaging methods including diffusion spectrum imaging, where crossing fibers are included in the model [44] have been shown to generate networks with a higher probability of long-distance connections [45]. For future studies, such advanced diffusion acquisition methods can be used to construct enhanced anatomical networks. Second, the FA value, which we used to weight the edge in white matter network, may not precisely reflect connectivity [46]. However, there is currently no formal consensus regarding selection of the edge weight which best describes the fiber tract connectivity and change in FA value probably reflect differences or damages in some aspects of connectivity. Third, due to limitations of the software package that we used for motion correction (FSL package), we could not apply rotation derived from subject head motion to the encoding vectors. This process will enhance the estimation of diffusion indices in future studies [46]. Finally, PiB-PET may not be sufficiently sensitive to detect soluble amyloid oligomers, diffuse amyloid plaques, or neurofibrillary tangle-predominant AD. Fourth, although we excluded patients with dementia with Lewy bodies and tau frontotemporal dementia using clinical criteria, patients with synuclein or tau pathologies might have been included in the present study.

This study shows that the approach of characterizing the brain as a network using DTI and graph theoretical analysis can provide new insights into how amyloid and vascular burdens affect white matter connectivity in patients with cognitive impairment.

\section{ACKNOWLEDGMENTS}

This study was supported by Basic Research Program through the National Research Foundation of Korea (NRF) funded by the Ministry of Education (NRF-2013R1A1A2065365), by the Korean Healthcare Technology R\&D Project Ministry for Health \& Welfare Affairs (HI10C2020 \& HIC120713), by the Korea Ministry of Environment (MOE) as the Environmental Health Action Program (2014001360002), by the KOSEF NRL program grant (MEST; 2011-0028333), by Samsung Medical Center (CRL-108011\&CRS110-14-1), and by the Converging Research Center Program through the Ministry of Science, ICT and Future Planning, Korea (2013K000338).

Authors' disclosures available online (http://www.jalz.com/disclosures/view.php?id=141623).

\section{SUPPLEMENTARY MATERIAL}

The supplementary material is available in the electronic version of this article: http://dx.doi.org/ 10.3233/JAD-141623.

\section{REFERENCES}

[1] Schneider JA, Arvanitakis Z, Leurgans SE, Bennett DA (2009) The neuropathology of probable Alzheimer disease and mild cognitive impairment. Ann Neurol 66, 200-208.

[2] Park JH, Seo SW, Kim C, Kim SH, Kim GH, Kim ST, Jeon S, Lee JM, Oh SJ, Kim JS, Choe YS, Lee KH, Shin JS, Kim $\mathrm{CH}$, Noh Y, Cho H, Yoon CW, Kim HJ, Ye BS, Ewers M, Weiner MW, Lee JH, Werring DJ, Na DL (2014) Effects of cerebrovascular disease and amyloid beta burden on cognition in subjects with subcortical vascular cognitive impairment. Neurobiol Aging 35, 254-260.

[3] Hedden T, Van Dijk KR, Shire EH, Sperling RA, Johnson KA, Buckner RL (2012) Failure to modulate attentional control in advanced aging linked to white matter pathology. Cereb Cortex 22, 1038-1051.

[4] Ye BS, Seo SW, Kim GH, Noh Y, Cho H, Yoon CW, Kim HJ, Chin J, Jeon S, Lee JM, Seong JK, Kim JS, Lee JH, Choe YS, Lee KH, Sohn YH, Ewers M, Weiner M, Na DL. (2014) Amyloid burden, cerebrovascular disease, brain atrophy, and cognition in cognitively impaired patients. Alzheimers Dement, doi: 10.1016/j.jalz.2014.04.521.

[5] Chao LL, Decarli C, Kriger S, Truran D, Zhang Y, Laxamana J, Villeneuve S, Jagust WJ, Sanossian N, Mack WJ, Chui HC, Weiner MW (2013) Associations between white matter hyperintensities and beta amyloid on integrity of projection, association, and limbic fiber tracts measured with diffusion tensor MRI. PLoS One 8, e65175.

[6] Lee DY, Fletcher E, Martinez O, Ortega M, Zozulya N, Kim J, Tran J, Buonocore M, Carmichael O, DeCarli C (2009) Regional pattern of white matter microstructural changes in normal aging, MCI, and AD. Neurology 73, 1722-1728.

[7] Sporns O, Tononi G, Kotter R (2005) The human connectome: A structural description of the human brain. PLoS Comput Biol 1, e42.

[8] Rubinov M, Sporns O (2010) Complex network measures of brain connectivity: Uses and interpretations. Neuroimage $\mathbf{5 2}$, 1059-1069.

[9] Seeley WW, Crawford RK, Zhou J, Miller BL, Greicius MD (2009) Neurodegenerative diseases target large-scale human brain networks. Neuron 62, 42-52.

[10] Raj A, Kuceyeski A, Weiner M (2012) A network diffusion model of disease progression in dementia. Neuron $\mathbf{7 3}$, 1204-1215.

[11] Zhou J, Gennatas ED, Kramer JH, Miller BL, Seeley WW (2012) Predicting regional neurodegeneration from the healthy brain functional connectome. Neuron 73, 1216-1227.

[12] Gong GL, He Y, Concha L, Lebel C, Gross DW, Evans AC, Beaulieu C (2009) Mapping anatomical connectivity patterns of human cerebral cortex using in vivo diffusion tensor imaging tractography. Cereb Cortex 19, 524-536.

[13] Latora V, Marchiori M (2001) Efficient behavior of smallworld networks. Phys Rev Let 87, 198701.

[14] He Y, Chen Z, Evans A (2008) Structural insights into aberrant topological patterns of large-scale cortical networks in Alzheimer's disease. J Neurosci 28, 4756-4766. 
[15] Agosta F, Sala S, Valsasina P, Meani A, Canu E, Magnani G, Cappa SF, Scola E, Quatto P, Horsfield MA, Falini A, Comi G, Filippi M (2013) Brain network connectivity assessed using graph theory in frontotemporal dementia. Neurology $\mathbf{8 1}$, 134-143.

[16] Lo CY, Wang PN, Chou KH, Wang J, He Y, Lin CP (2010) Diffusion tensor tractography reveals abnormal topological organization in structural cortical networks in Alzheimer's disease. J Neurosci 30, 16876-16885.

[17] Heringa SM, Reijmer YD, Leemans A, Koek HL, Kappelle LJ, Biessels GJ, Utrecht Vascular Cognitive Impairment Study G (2014) Multiple microbleeds are related to cerebral network disruptions in patients with early Alzheimer's disease. J Alzheimers Dis 38, 211-221.

[18] McKhann G, Drachman D, Folstein M, Katzman R, Price D, Stadlan EM (1984) Clinical diagnosis of Alzheimer's disease: Report of the NINCDS-ADRDA Work Group under the auspices of Department of Health and Human Services Task Force on Alzheimer's Disease. Neurology 34, 939-944.

[19] Erkinjuntti T, Inzitari D, Pantoni L, Wallin A, Scheltens P, Rockwood K, Roman GC, Chui H, Desmond DW (2000) Research criteria for subcortical vascular dementia in clinical trials. J Neural Transm Suppl 23-30.

[20] Seo SW, Cho SS, Park A, Chin J, Na DL (2009) Subcortical vascular versus amnestic mild cognitive impairment: Comparison of cerebral glucose metabolism. J Neuroimaging 19, 213-219.

[21] Fazekas F, Kleinert R, Offenbacher H, Schmidt R, Kleinert G, Payer F, Radner H, Lechner H (1993) Pathologic correlates of incidental MRI white matter signal hyperintensities. Neurology 43, 1683-1689.

[22] Wardlaw JM, Smith EE, Biessels GJ, Cordonnier C, Fazekas F, Frayne R, Lindley RI, O'Brien JT, Barkhof F, Benavente OR, Black SE, Brayne C, Breteler M, Chabriat H, Decarli C, de Leeuw FE, Doubal F, Duering M, Fox NC, Greenberg S, Hachinski V, Kilimann I, Mok V, Oostenbrugge R, Pantoni L, Speck O, Stephan BC, Teipel S, Viswanathan A, Werring D, Chen C, Smith C, van Buchem M, Norrving B, Gorelick PB, Dichgans M, STandards for ReportIng Vascular changes on nEuroimaging (STRIVE, v1) (2013) Neuroimaging standards for research into small vessel disease and its contribution to ageing and neurodegeneration. Lancet Neurol 12, 822-838.

[23] Seo SW, Im K, Lee JM, Kim YH, Kim ST, Kim SY, Yang DW, Kim SI, Cho YS, Na DL (2007) Cortical thickness in singleversus multiple-domain amnestic mild cognitive impairment. Neuroimage 36, 289-297.

[24] Tzourio-Mazoyer N, Landeau B, Papathanassiou D, Crivello F, Etard O, Delcroix N, Mazoyer B, Joliot M (2002) Automated anatomical labeling of activations in SPM using a macroscopic anatomical parcellation of the MNI MRI singlesubject brain. Neuroimage 15, 273-289.

[25] Collins DL, Neelin P, Peters TM, Evans AC (1994) Automatic 3D intersubject registration of MR volumetric data in standardized Talairach space. J Comput Assist Tomogr 18, 192-205.

[26] Mori S, Crain BJ, Chacko VP, van Zijl PCM (1999) Threedimensional tracking of axonal projections in the brain by magnetic resonance imaging. Ann Neurol 45, 265-269.

[27] Wang R, Beener T, Sorensen AG, Wedeen VJ (2007) Diffusion toolkit: A software package for diffusion imaging data processing and tractography. Proc Int Soc Magn Reson Med 15, 3720 .
[28] Im K, Paldino MJ, Poduri A, Sporns O, Grant PE (2014) Altered white matter connectivity and network organization in polymicrogyria revealed by individual gyral topology-based analysis. Neuroimage 86, 182-193.

[29] Guevara P, Poupon C, Riviere D, Cointepas Y, Descoteaux M, Thirion B, Mangin JF (2011) Robust clustering of massive tractography datasets. Neuroimage 54, 1975-1993.

[30] Shu N, Liu Y, Li K, Duan Y, Wang J, Yu C, Dong H, Ye J, He Y (2011) Diffusion tensor tractography reveals disrupted topological efficiency in white matter structural networks in multiple sclerosis. Cereb Cortex 21, 2565-2577.

[31] Beaulieu C (2002) The basis of anisotropic water diffusion in the nervous system - a technical review. NMR Biomed 15, 435-455.

[32] Watts DJ, Strogatz SH (1998) Collective dynamics of 'smallworld' networks. Nature 393, 440-442.

[33] Onnela JP, Saramaki J, Kertesz J, Kaski K (2005) Intensity and coherence of motifs in weighted complex networks. Phys Rev E Stat Nonlin Soft Matter Phys 71, 065103.

[34] Maslov S, Sneppen K (2002) Specificity and stability in topology of protein networks. Science 296, 910-913.

[35] Blondel VD, Guillaume JL, Lambiotte R, Lefebvre E. (2008) Fast unfolding of communities in large networks. J Stat Mech Theory Exp, P10008.

[36] Kang Y, Na DL (2003) Seoul Neuropsychological Screening Battery. Human Brain Research \& Consulting, Incheon.

[37] Ahn HJ, Chin J, Park A, Lee BH, Suh MK, Seo SW, Na DL (2010) Seoul Neuropsychological Screening Batterydementia version (SNSB-D): A useful tool for assessing and monitoring cognitive impairments in dementia patients. J Korean Med Sci 25, 1071-1076.

[38] Tijms BM, Moller C, Vrenken H, Wink AM, de Haan W, van der Flier WM, Stam CJ, Scheltens P, Barkhof F (2013) Singlesubject grey matter graphs in Alzheimer's disease. PLoS One 8, e58921.

[39] Franklin RJ, Ffrench-Constant C (2008) Remyelination in the CNS: From biology to therapy. Nat Rev Neurosci 9, 839-855.

[40] Iadecola C (2013) The pathobiology of vascular dementia. Neuron 80, 844-866.

[41] Tijms BM, Wink AM, de Haan W, van der Flier WM, Stam CJ, Scheltens P, Barkhof F (2013) Alzheimer's disease: Connecting findings from graph theoretical studies of brain networks. Neurobiol Aging 34, 2023-2036.

[42] Reijmer YD, Leemans A, Caeyenberghs K, Heringa SM, Koek HL, Biessels GJ, Utrecht Vascular Cognitive Impairment Study G (2013) Disruption of cerebral networks and cognitive impairment in Alzheimer disease. Neurology 80, 1370-1377.

[43] Tullberg M, Fletcher E, DeCarli C, Mungas D, Reed BR, Harvey DJ, Weiner MW, Chui HC, Jagust WJ (2004) White matter lesions impair frontal lobe function regardless of their location. Neurology 63, 246-253.

[44] Wedeen VJ, Wang RP, Schmahmann JD, Benner T, Tseng WY, Dai G, Pandya DN, Hagmann P, D'Arceuil H, de Crespigny AJ (2008) Diffusion spectrum magnetic resonance imaging (DSI) tractography of crossing fibers. Neuroimage 41, 1267-1277.

[45] Zalesky A, Fornito A, Harding IH, Cocchi L, Yucel M, Pantelis C, Bullmore ET (2010) Whole-brain anatomical networks: Does the choice of nodes matter? Neuroimage 50, 970-983.

[46] Jones DK, Knosche TR, Turner R (2013) White matter integrity, fiber count, and other fallacies: The do's and don'ts of diffusion MRI. Neuroimage 73, 239-254. 